\title{
Loin des murs
}

Entretien mené par Rym Khene

\section{Fatima Chafaa}

\section{OpenEdition}

\section{Journals}

Édition électronique

URL : https://journals.openedition.org/coma/5252

DOI : $10.4000 /$ coma.5252

ISSN : 2275-1742

Éditeur

Institut des textes \& manuscrits modernes (ITEM)

Référence électronique

Fatima Chafaa, "Loin des murs », Continents manuscrits [En ligne], 14 | 2020, mis en ligne le 15 mars 2020, consulté le 13 janvier 2023. URL : http://journals.openedition.org/coma/5252 ; DOI : https:// doi.org/10.4000/coma.5252

Ce document a été généré automatiquement le 13 janvier 2023.

\section{(2) $(\mathcal{Q} \Theta \Theta$}

Creative Commons - Attribution - Pas d'Utilisation Commerciale - Pas de Modification 4.0 International - CC BY-NC-ND 4.0

https://creativecommons.org/licenses/by-nc-nd/4.0/ 


\title{
Loin des murs
}

\author{
Entretien mené par Rym Khene
}

\section{Fatima Chafaa}

Cet entretien a été réalisé en septembre 2019.

Rym Khene : Votre projet, "Loin des murs de Takamra », est le récit de la découverte de ce village. À travers une installation photographique, vous mettez en scène des photos de famille, comme pour rendre visible une généalogie et raconter l'histoire familiale pour proposer un récit de l'histoire algérienne. En utilisant le médium photographique, vous proposez également une réflexion sur l'utilisation de cet outil et invitez le spectateur à réfléchir sur le rôle de la photographie.

Avec cette installation, vous mettez en scène des portraits de famille réalisés par votre oncle photographe Bachir Chafaa. Tous sont nés à Takamra, et tous ont été déracinés. Ces photographies sont emboîtées dans la pierre, incrustées presque, pour rendre vivants la pierre, la terre, l'histoire, et bien sûr les visages et les corps.

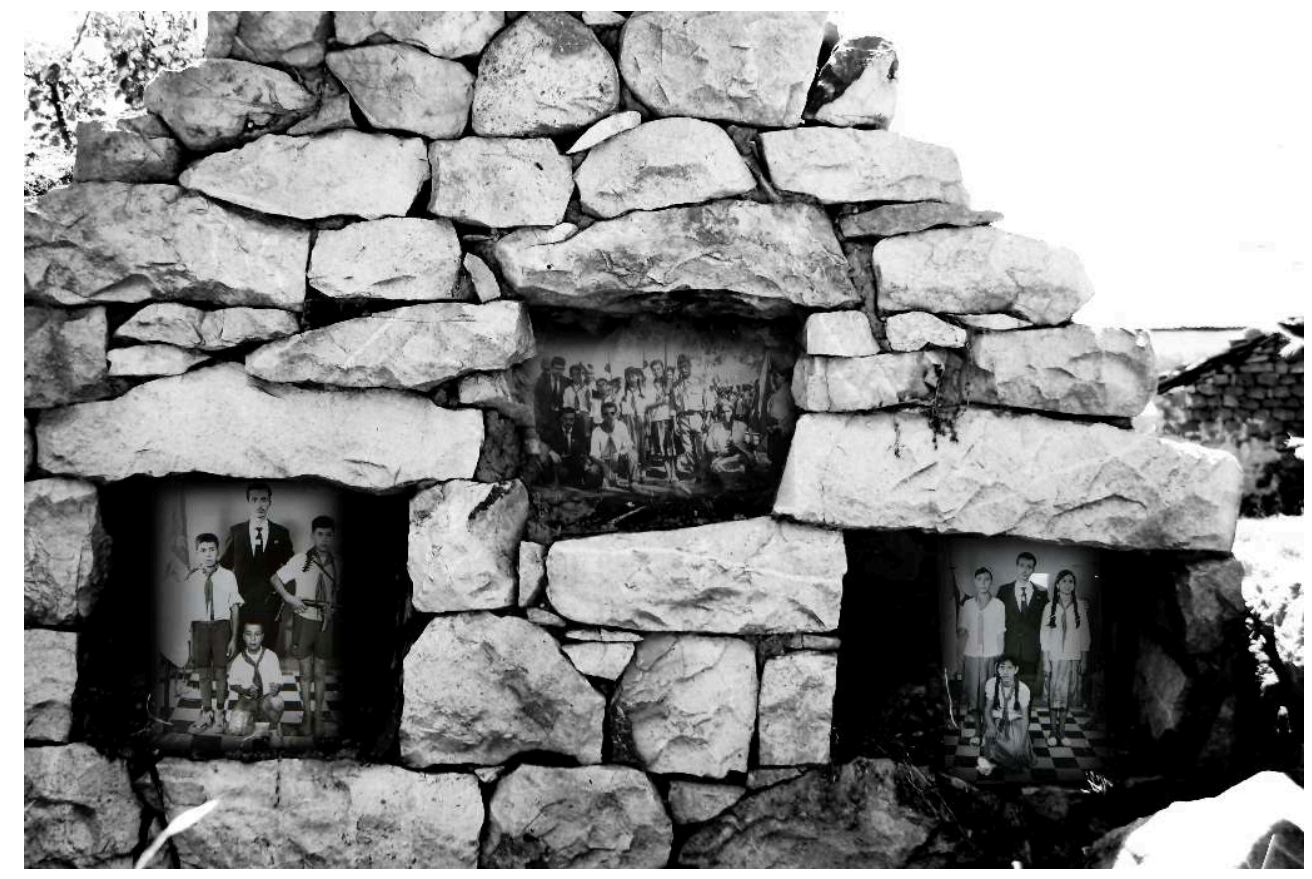


Figure 1 : Oncle Ali

Détail de l'installation «Loin des murs », 2010. Photos d'archives familiales mises en scène par Fatima Chafaa.

(c) Fatima Chafaa

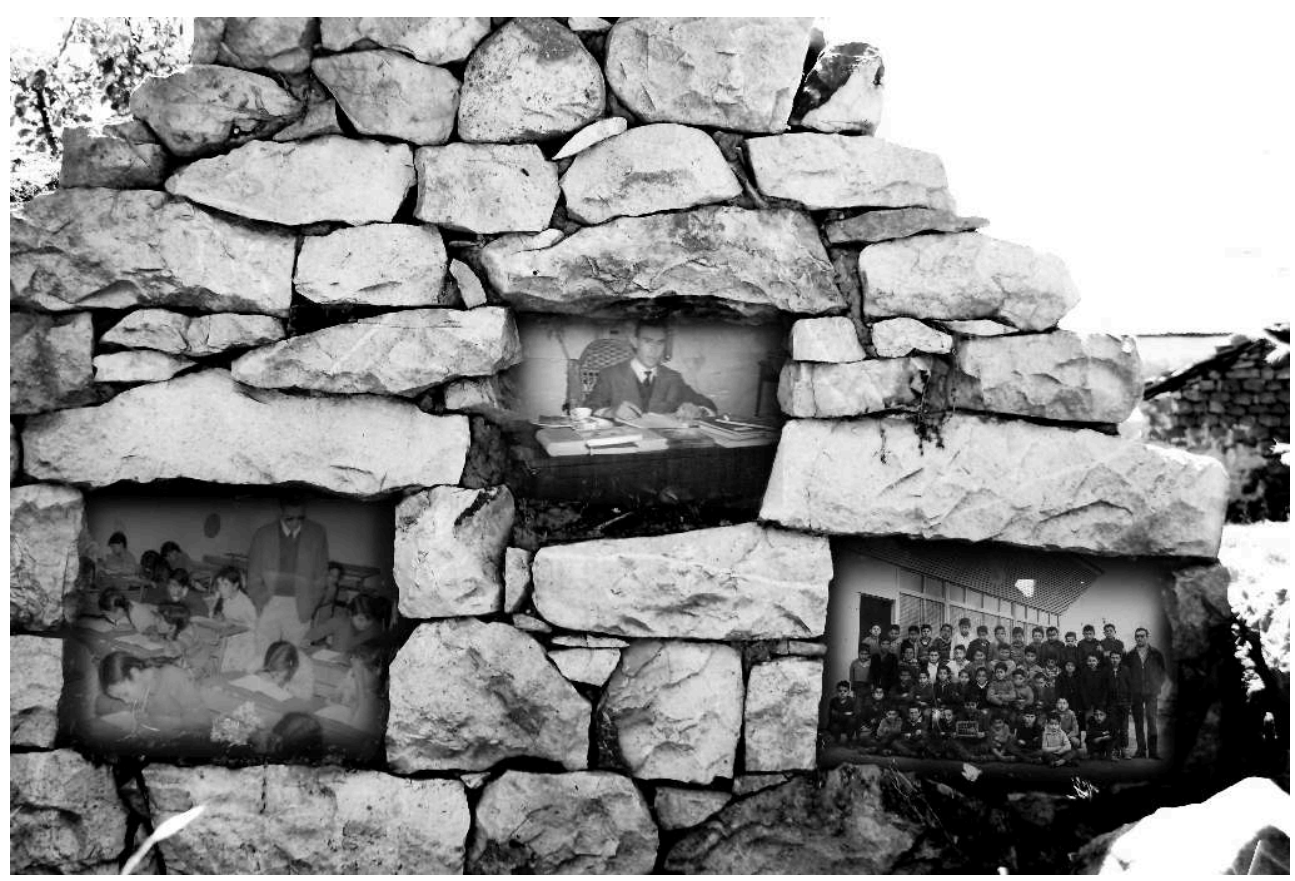

Figure 2 : Oncle Faudile

Détail de l'installation « Loin des murs », 2010. Photos d'archives familiales mises en scène par Fatima Chafaa.

(c) Fatima Chafaa

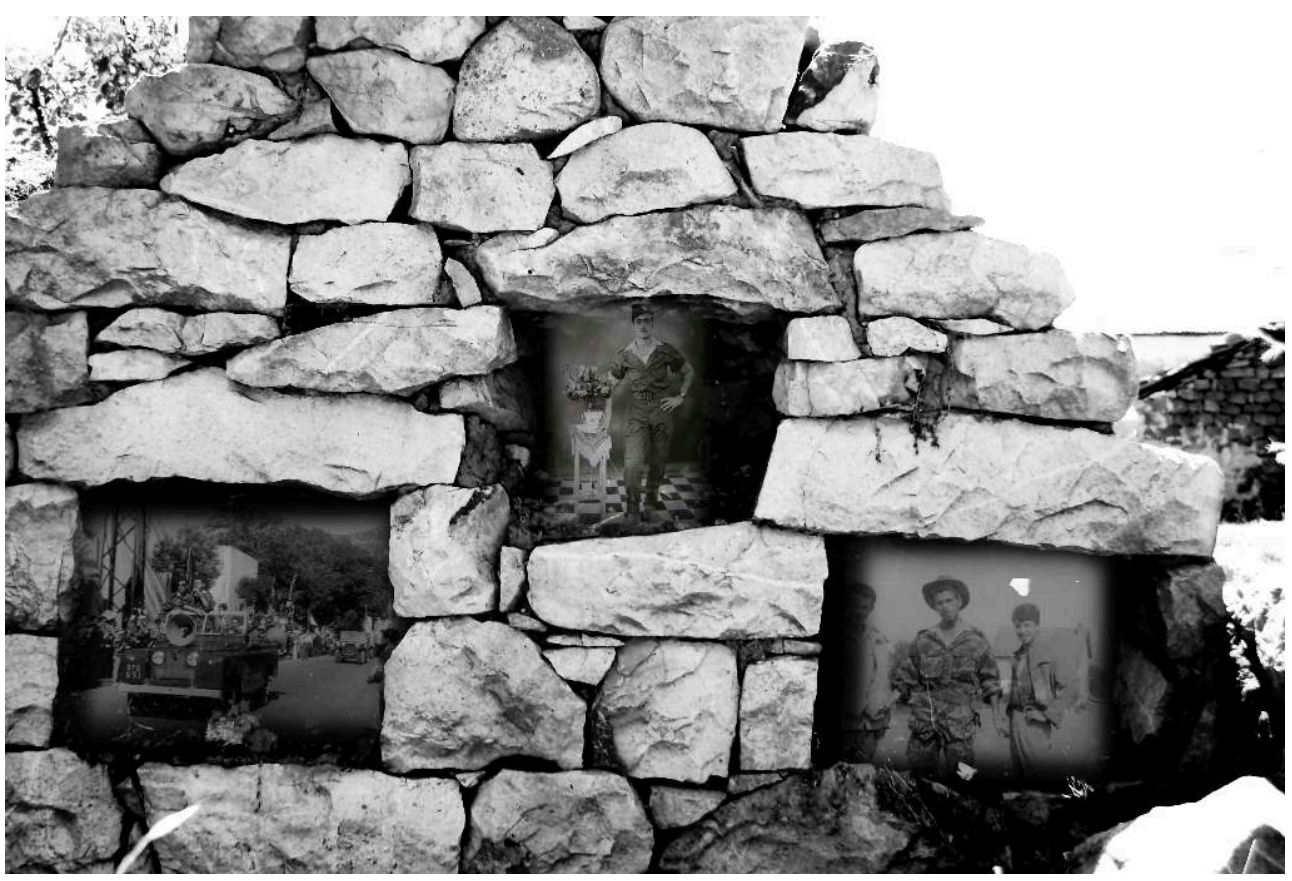


Figure 3 : Oncle Rachid

Détail de l'installation « Loin des murs », 2010. Photos d'archives familiales mises en scène par Fatima Chafaa.

(c) Fatima Chafaa

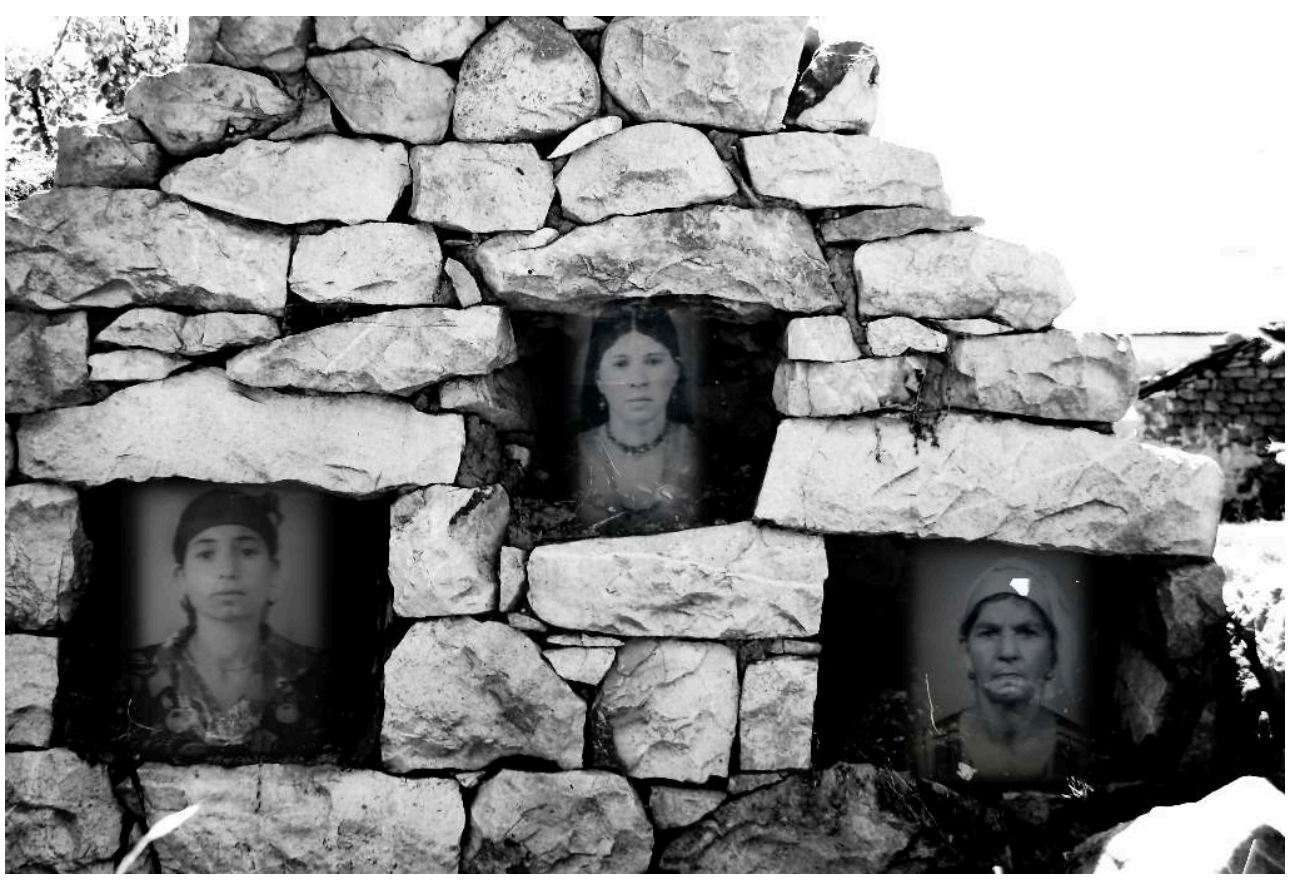

Figure 4 : Tante Djida

Détail de l'installation «Loin des murs », 2010. Photos d'archives familiales mises en scène par Fatima Chafaa.

(c) Fatima Chafaa

Connaissez-vous I'histoire «photographique » de Bachir Chafaa? Dans quelles conditions a-t-il exercé son métier de photographe? Quel genre de photos faisait-il ? Était-ce essentiellement des portraits ? Photographiait-il régulièrement les membres de sa famille ou bien était-ce exceptionnel ? Parlait-il du métier de photographe et de l'importance de la photographie?

Fatima Chafaa: Mon oncle Bachir est né en 1931, à Takamra, village situé sur les hauteurs de la Kabylie. Très jeune, il rejoint mon père en France, où il apprend la photographie. Je ne sais pas dans quelles conditions, ni avec qui. Toujours est-il, qu'en 1962, après l'indépendance, il rentre en Algérie et s'installe à Aïn Benian, à $16 \mathrm{~km}$ de la capitale. Aïn Benian est composée d'une grande communauté kabyle qui y trouve refuge après les bombardements de la Kabylie par l'armée française en 1956.

Dès son arrivée, mon oncle a commencé à prendre en photo la vie quotidienne des habitants du village de Takamra désormais installés à Aïn Benian. Il était en général spontané dans ses prises de vue, très discret lorsqu'il faisait des photos. Parfois, il tendait aussi vers des mises en scène familiales, dont la forme était très simple : il aimait rassembler les membres de la famille autour d'un café et les photographier. 


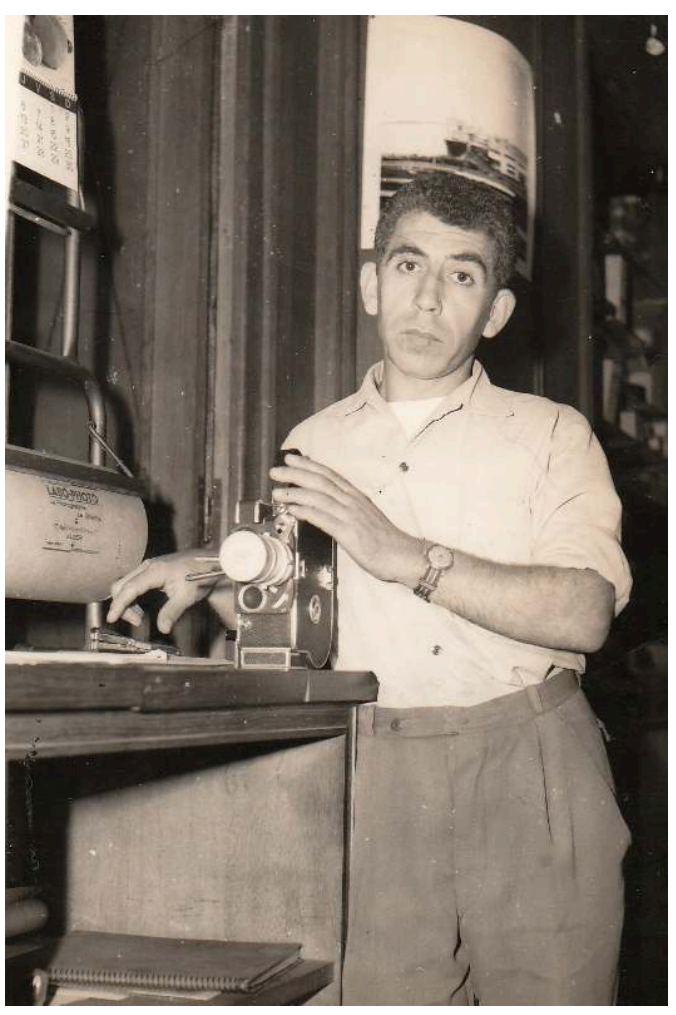

Figure 5 : Oncle Bachir

Bachir Chafaa, photographe. Autoportrait.

(C) Archives personnelles de Fatima Chafaa. Date incertaine (1963-1970)

Son appareil photo toujours sur lui, il immortalisait ainsi les événements familiaux. Quelques membres de la famille ont marché sur ces traces, parmi eux mon oncle Mouloud qui travaillait au cinéma Soummam à Aïn Benian ainsi que deux de mes cousins qui ont fondé des studios photographiques très connus à Aïn Benian, le studio-photo Marouf et Akram. Dans les années 1970, mon oncle Bachir devient photographe pour l'Armée nationale populaire, jusqu'à sa retraite. Il meurt en 2013. 


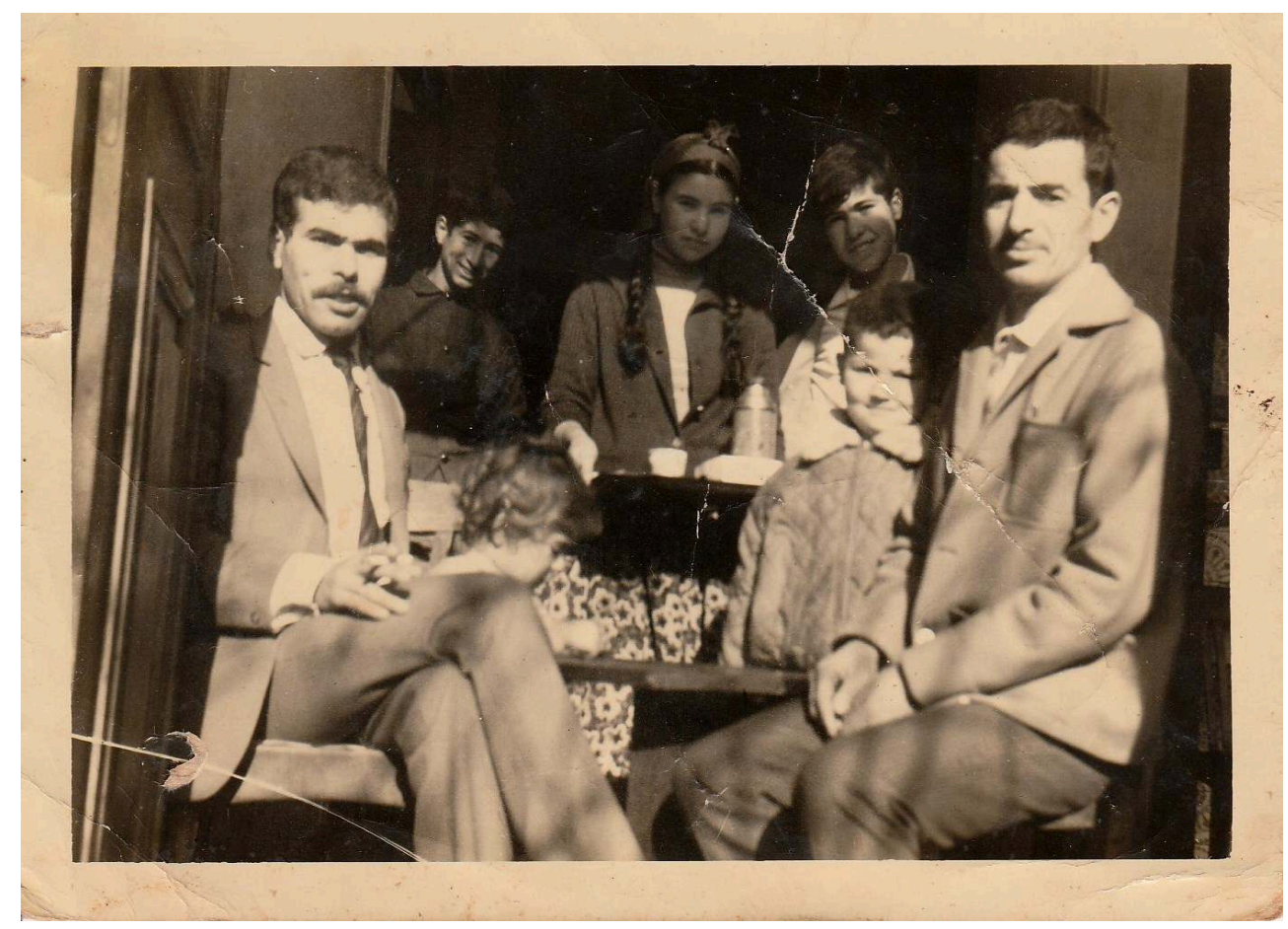

Figure 6

Mise en scène familiale. Photographie prise par Bachir Chafaa.

(C) Archives personnelles de Fatima Chafaa. Date incertaine (1963-1980)

$\mathrm{RK}$ : Pourquoi avoir placé les photographies dans la pierre?

FC : Le mur en pierre est un mur de la maison de mes grands-parents à Takamra. C'est un mur qui porte une légende de la région.

Takamra, village perché, sur les hauteurs de la Kabylie, région montagneuse de l'Algérie, est aussi le village où naissent et grandissent mes parents. Il est l'un des trois villages équidistants qui constituent le Arch (ou tribu) maraboutique des Ath Sidi Moussa.

Selon la mythologie locale, les trois filles de notre aïeul, le saint marabout Sidi Moussa, fondèrent les trois villages à qui elles donnèrent leurs prénoms, Takamra, Fetala, Taourirt. C'est pour cela que, jusqu'à ce jour, dans la salle principale de chaque maison de ces trois villages, à l'endroit où l'on garde le feu du foyer, trois trous rectangulaires sont creusés dans le mur qui fait face à la porte. C'est en quelque sorte l'autel des trois saintes, symboles de fécondité et de prospérité.

En 1956, pour cause de son soutien à la résistance algérienne, l'armée coloniale française déclare nos trois villages zone interdite, elle les rase et décime leurs populations. Je n'ai pas de traces visuelles, ou d'archives de cette période-là, à part une photographie de mon grand-père et de son frère, condamnés par l'armée française ${ }^{1}$. 


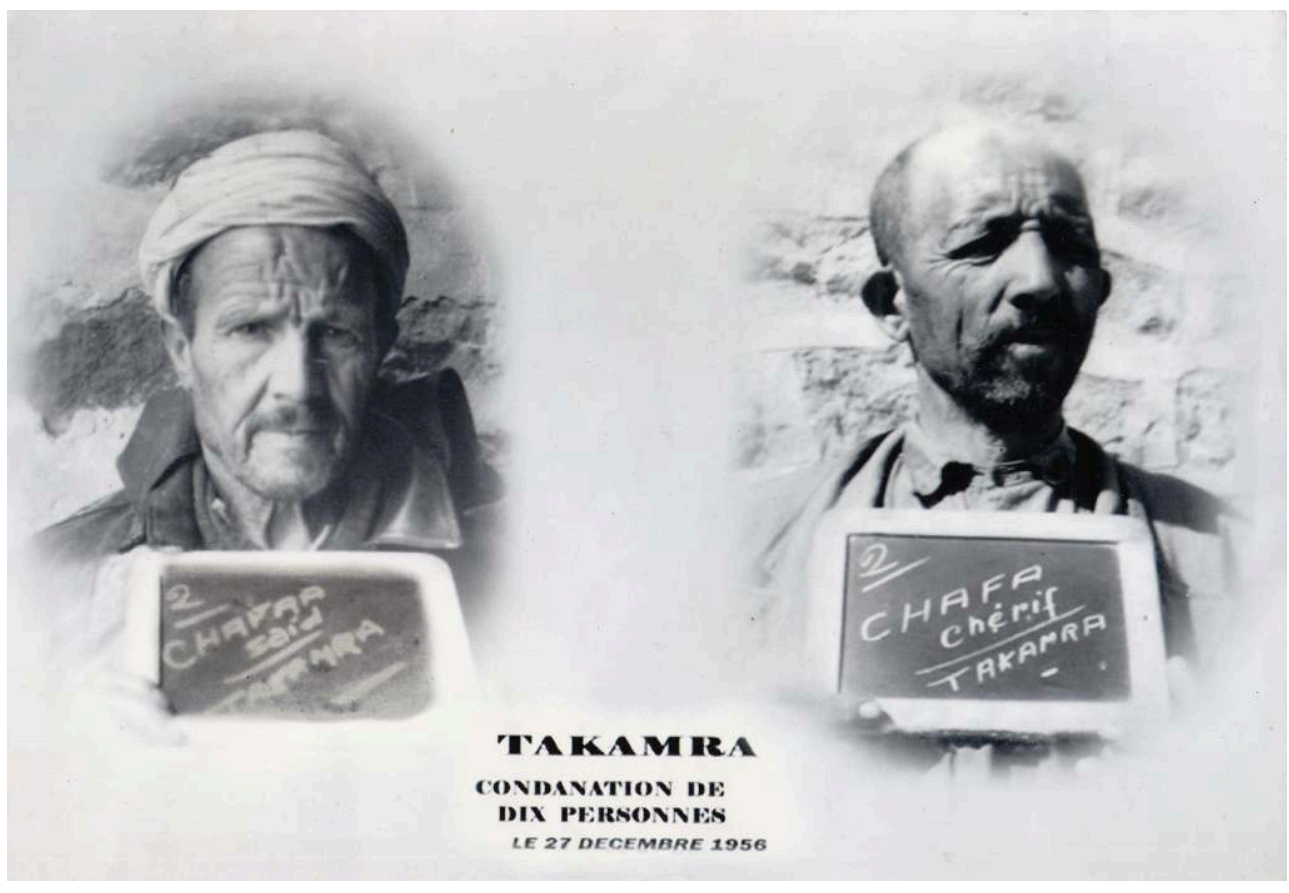

Figure 7

Le grand-père de Fatima Chafaa et son frère condamnés par l'Armée française en 1956. La photographie a été retrouvée en 1962 au poste de la commune d'Adekar (Wilaya de Bejaïa). Ils seront libérés et s'installeront à Aïn Benian (anciennement appelé Guyotville).

(c) Fatima Chafaa

À l'indépendance de l'Algérie en 1962, le gouvernement propose une aide financière aux villageois déracinés pour un retour sur leurs terres, mais mes parents ne sont jamais retournés vivre à Takamra.

Pour moi, placer les photos dans la pierre, c'est raconter à ces pierres ce que sont devenus ces fiers paysans et leurs familles. Chassés de leur terre natale, ils se sont retrouvés sur les routes de l'errance. Une chose est pourtant sûre ; ils n'ont jamais renié leur terre et leur culture. Je trouve qu'il est important de transmettre cela aux générations futures, de leur faire découvrir des traces de leur histoire, de leur passé.

RK : Les murs pour symboliser la solidité d'un héritage, d'une histoire, d'une mythologie? Lorsque je regarde la photo, j'ai l'impression que ces visages vivent sur une frontière. Cette frontière n'est pas forcément géographique, puisque, comme vous l'indiquez, dans le titre de votre installation, nous sommes «loin des murs", mais il y a un jeu temporel et une multiplicité géographique, comme si, en exhumant ces photos d'archives, vous proposiez d'interroger une narration, une histoire, mais d'une façon nouvelle, comme si la narration « classique » ne suffisait pas.

FC : Malgré notre vie citadine, nos parents ont toujours tenu à nous transmettre notre culture amazighe, et nous ont toujours parlé de leur village Takamra et de ses coutumes ancestrales. D'ailleurs, durant ma jeunesse, ma mère me confectionnait toujours des robes kabyles pour la fête de l'Aïd.

En souvenir de son village natal, mon père a planté des figuiers de Barbarie dans son jardin à Aïn Benian, comme s'il voulait garder près de lui ses souvenirs.

C'est en 2006 que j'ai ressenti, pour la première fois, le besoin d'aller à Takamra, de me rapprocher de la culture de mes parents, de parler à ces murs, de leur raconter ce 
que les villageois déracinés étaient devenus loin de leur protection, et leur dire que même en allant dans un autre village, ils avaient transmis leur héritage.

Mon installation photographique se compose d'une photo que j'ai prise du mur de la maison de mes grands-parents. J'y ai placé des photos prises par mon oncle Bachir que j'ai récupérées d'un album familial. Dans le mur en pierre, j'ai également placé les photos des membres de ma famille nés à Takamra et qui vivent, ou vécurent à Alger ou en France. J'ai essayé de raconter des petites histoires à ce mur de pierre qui les a protégés, et de montrer ce qu'ils étaient devenus loin de lui.

Raconter par exemple l'histoire de mon père, longtemps émigré en France, où il était ouvrier dans une tannerie et qui, lors d'un accident du travail, a perdu un doigt. Ou encore cette "photo mur " représentant l'histoire de ma mère Ouardhia qui quitta son village natal à l'âge de 17 ans, ne parlant que sa langue maternelle, le kabyle... Peut-être que raconter son histoire aux autres passe d'abord par le geste de la raconter à la pierre et à la terre.

RK : Quelle a donc été votre démarche, votre réflexion?

FC : À un certain moment, j'ai voulu faire le lien entre deux générations: une génération qui a vécu ce passé (à travers les photos de mon oncle) et une autre, à qui on a transmis cet héritage culturel (à travers les miennes). Pour cela, il a fallu aller vers Takamra, et se rendre au mausolée de Sidi Moussa, qui se trouve dans le village de sa fille Fatala. En 2006, je l'ai découvert pour la première fois, en compagnie de ma mère. Là, j'ai vu que la maison de mes parents était en ruines. J'ai réalisé des photographies en me concentrant surtout sur le mur principal de la maison où se trouvent les trois trous. Ainsi, j'ai commencé à me remémorer la légende que me racontait mon père... je voulais, je pense, leur rendre hommage.

RK: Quelle est la différence pour vous, entre une exposition photographique et une installation? Que permet l'une que ne permet pas l'autre?

FC: Une installation est une œuvre à trois dimensions, elle me permet d'inclure l'environnement et des éléments d'archives qui interagissent avec leur environnement. Pour ce projet, une exposition de photographies aurait figé les images.

Le travail s'est fait essentiellement dans mon atelier à Aïn Benian. J'ai utilisé l'album photo familial, et surtout les photos prises par mon oncle Bachir.

L'installation photographique a été exposée à Alger à l'occasion du $2^{\mathrm{e}}$ Festival national de la photographie au Musée national d'art moderne et contemporain (MAMA), en Suisse (AB galerie) et à Moscou (Family Unity is the Unity of the world: Ekaterinbourg Art Foundation).

\section{NOTES}

1. Pour le récit du déplacement de la famille Chafaa vers Aïn Benian, voir le film réalisé par Fatima Chafaa : https://vimeo.com/user89809824. 
INDEX

Mots-clés : photographie, archives, histoire photographique, installation, Algérie Index géographique : Algérie

\section{AUTEUR}

\section{FATIMA CHAFAA}

FATIMA CHAFAA est née aux environs d'Alger. Après une formation en agronomie, elle rejoint ses cousins et ses oncles au sein du studio photo familial. En 2000, elle intègre l'école des Beaux-Arts d'Alger (spécialité peinture). Son travail mêle photographie, installation et arts plastiques. À travers une approche souvent intime, elle interroge l'histoire, les normes sociales et sociétales. Elle expose régulièrement en Algérie et à l'étranger. https://chafaafatima.jimdofree.com/ 